\section{Reply to V. Turan et al}

We appreciate the interest of Turan et $\mathrm{al}^{1}$ in our systematic review and individual patient-level data metaanalysis of randomized controlled trials (RCTs) that explored the role of temporary ovarian suppression with gonadotropin-releasing hormone agonists (GnRHa) during chemotherapy as a strategy to preserve ovarian function and fertility in premenopausal patients with breast cancer. ${ }^{2}$

The authors consider the exclusion of RCTs in patients with hematologic malignancies as a limitation of our analysis; however, the decision to focus only on breast cancer RCTs was based on both methodological and clinical reasons. First, only 154 patients were included in four RCTs for hematologic malignancies compared with 13 breast cancer RCTs that included 1,581 women. ${ }^{3,4}$ Second, women with hematologic malignancies or breast cancer included in these RCTs were quite different in terms of both age at diagnosis (ie, approximately 25 years for hematologic malignancies, 40 years for breast cancer) and chemotherapy regimens used (ie, with high [eg, conditioning regimens for hematopoietic stem-cell transplantation] or low [eg, doxorubicin, bleomycin, vinblastine, and dacarbazine] gonadotoxic potential for hematologic malignancies, with intermediate gonadotoxicity [eg, cyclophosphamide-based regimens] for breast cancer), ${ }^{3,4}$ With consideration that both the numerical imbalance and the fact that age as well as type of chemotherapy are the most important factors affecting the risk of premature ovarian insufficiency (POI), ${ }^{5}$ the RCTs that investigated GnRHa use during chemotherapy in women with hematologic malignancies or breast cancer should not be mixed. The use of oral contraceptives during chemotherapy and oncologic follow-up in several women treated for hematologic malignancies adds complexity in the interpretation of these RCTs. ${ }^{3}$

Turan et $\mathrm{al}^{1}$ raise additional concerns about the impossibility to collect individual patient-level data from all the available breast cancer RCTs, the lack of significant reduction in amenorrhea risk 1 year after chemotherapy completion, and the absence of data on antimullerian hormone (AMH) levels.

As we have acknowledged, ${ }^{2}$ despite our efforts in trying to obtain data from all RCTs, we were able to collect individual patient-level data from the five major published RCTs, including $55.2 \%$ of the potentially eligible population (873 of 1,581 ). However, there was no disproportional representation of positive and negative RCTs included or not in our analysis ${ }^{2,4}$ (Table 1).

In addition and of note, our prior meta-analysis that was based on abstracted data from all the available breast cancer RCTs except for the OPTION (Ovarian
Protection Trial in Premenopausal Breast Cancer Patients) study (a positive trial, which was not available at the time $)^{6}$ showed similar results to our present analysis ${ }^{2,4}$ (Table 1 ). The only exception is represented by 1 -year amenorrhea for which a protective effect that favors GnRHa administration during chemotherapy was observed only when a larger number of RCTs was included. ${ }^{2,4}$ Of note, our findings showing that approximately $15 \%$ of women resume menstrual function $>1$ year after chemotherapy also support the expert opinion-based definition of chemotherapyinduced $\mathrm{POI}$ to be assessed not earlier than 2 years after the end of cytotoxic therapy.

We acknowledged the lack of AMH data as a limitation to assess the gonadal protection of GnRHa use. ${ }^{2}$ However, three of the five included RCTs defined POI on the basis of not only post-treatment amenorrhea but also postmenopausal levels of other ovarian function parameters, such as follicle-stimulating hormone and estradiol. Indeed, a composite end point that included menstrual function and follicle-stimulating hormone levels $>25 \mathrm{IU} / \mathrm{L}$ is the currently adopted definition of POI. ${ }^{7,8}$ Moreover, although AMH plays an important role in predicting oocyte yield after controlled ovarian stimulation, recent studies have questioned its value as a marker of chemotherapy-induced $\mathrm{POI}$ and infertility by showing that both menstrual function resumption and post-treatment pregnancies can be observed in women with low AMH levels. ${ }^{3,9}$

We agree that the data on post-treatment pregnancies are limited and that temporary ovarian suppression with GnRHa during chemotherapy should not preclude the access to oocyte/embryo cryopreservation. However, on the basis of the observed significantly higher pregnancy rate for women treated with $\mathrm{GnRHa},{ }^{2}$ this strategy can be offered as an adjunct for patients after cryopreservation techniques to increase the chances of natural conception as well for those who do not have access to assisted reproductive options.

Moreover, preservation of ovarian function and avoidance of its negative consequences and associated symptoms may represent another important goal for premenopausal patients with breast cancer irrespective of their pregnancy desire and age at diagnosis. On the basis of our findings and as recommended by current guidelines (including those recently updated by ASCO), ,,10,11 temporary ovarian suppression with GnRHa during chemotherapy can now be considered as a strategy to reduce the likelihood of $\mathrm{POI}$ for premenopausal women in whom continued ovarian function is desirable. Future wellconducted in vitro and in vivo animal experiments in species other than rodents should be encouraged to explain the protective mechanism of action of $\mathrm{GnRHa}$. 
TABLE 1. Comparison of Meta-Analyses of RCTs on the Role of Temporary Ovarian Suppression With GnRHas During Chemotherapy in Premenopausal Patients With Breast Cancer

Meta-Analysis

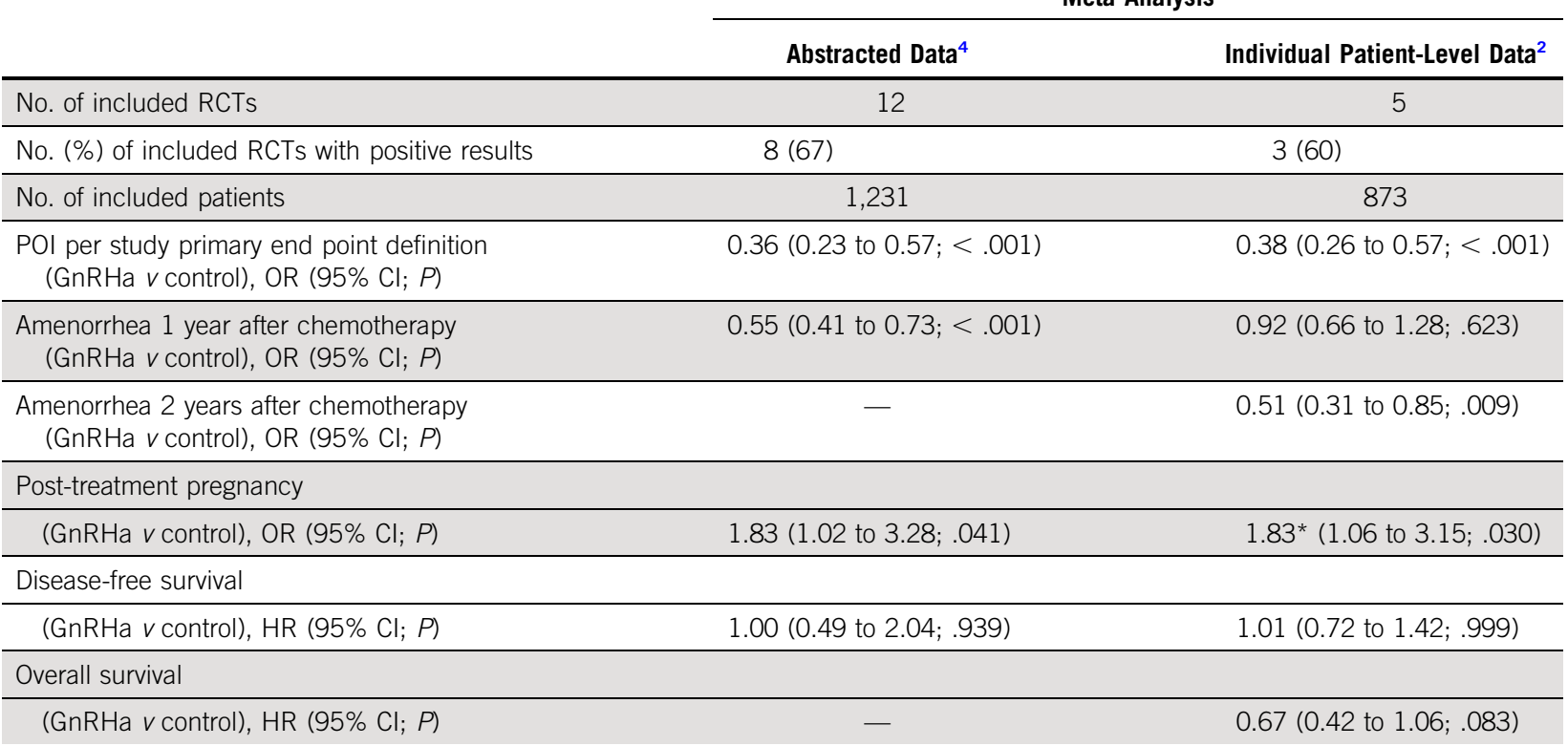

NOTE. Comparison between the largest meta-analysis on the basis of abstracted data and the current meta-analysis on the basis of individual patient-level data of RCTs that explored the role of temporary ovarian suppression with GnRHa during chemotherapy as a strategy to preserve ovarian function and fertility in premenopausal patients with breast cancer.

Abbreviations: GnRHa, gonadotropin-releasing hormone agonist; $\mathrm{HR}$, hazard ratio; OR, odds ratio; POI, premature ovarian insufficiency; RCT, randomized controlled trial.

*Incidence rate ratio.

\section{Matteo Lambertini, MD}

Institut Jules Bordet and Université Libre de Bruxelles, Brussels, Belgium

\section{Ann H. Partridge, MD}

Dana-Farber Cancer Institute, Boston, MA

\section{Lucia Del Mastro}

Ospedale Policlinico San Martino and University of Genoa, Genoa, Italy

AUTHORS' DISCLOSURES OF POTENTIAL CONFLICTS OF INTEREST AND DATA AVAILABILITY STATEMENT

Disclosures provided by the authors and data availability statement (if applicable) are available with this article at DOI https://doi.org/ $10.1200 / J C 0.18 .00630$

\section{REFERENCES}

1. Turan V, Bedoschi G, Rodriguez-Wallberg K, et al: Utility of gonadotropinreleasing hormone agonists for fertility preservation: Lack of biologic basis and the need to prioritize proven methods. J Clin Oncol 37:84-86, 2019

2. Lambertini M, Moore HCF, Leonard RCF, et al: Gonadotropin-releasing hormone agonists during chemotherapy for preservation of ovarian function and fertility in premenopausal patients with early breast cancer: A systematic review and meta-analysis of individual patient-level data. J Clin Oncol 36:1981-1990, 2018

3. Demeestere I, Brice P, Peccatori FA, et al: No evidence for the benefit of gonadotropin-releasing hormone agonist in preserving ovarian function and fertility in lymphoma survivors treated with chemotherapy: Final long-term report of a prospective randomized trial. J Clin Oncol 34: 2568-2574, 2016

4. Lambertini M, Ceppi M, Poggio F, et al: Ovarian suppression using luteinizing hormone-releasing hormone agonists during chemotherapy to preserve ovarian function and fertility of breast cancer patients: A metaanalysis of randomized studies. Ann Oncol 26:2408-2419, 2015

5. Lambertini M, Campbell C, Bines J, et al: Adjuvant anti-HER2 therapy, treatment-related amenorrhea, and survival in premenopausal HER2positive early breast cancer patients. J Natl Cancer Inst 10.1093/jnci/ djy094 [epub ahead of print on June 5, 2018]

6. Leonard RCF, Adamson DJA, Bertelli G, et al: GnRH agonist for protection against ovarian toxicity during chemotherapy for early breast cancer: The Anglo Celtic Group OPTION trial. Ann Oncol 28:1811-1816, 2017

7. Paluch-Shimon S, Pagani O, Partridge AH, et al: ESO-ESMO 3rd international consensus guidelines for breast cancer in young women (BCY3). Breast 35:203-217, 2017

8. ESHRE Writing Group on POI: ESHRE guideline: Management of women with premature ovarian insufficiency. Hum Reprod 31:926-937, 2016

9. Steiner AZ, Pritchard D, Stanczyk FZ, et al: Association between biomarkers of ovarian reserve and infertility among older women of reproductive age. JAMA 318:1367-1376, 2017

10. Lambertini M, Cinquini M, Moschetti I, et al: Temporary ovarian suppression during chemotherapy to preserve ovarian function and fertility in breast cancer patients: A GRADE approach for evidence evaluation and recommendations by the Italian Association of Medical Oncology. Eur J Cancer 71:25-33, 2017

11. Oktay $\mathrm{K}$, Harvey $\mathrm{BE}$, Partridge $\mathrm{AH}$, et al: Fertility preservation in patients with cancer: ASCO clinical practice guideline update. J Clin Oncol 36: 1994-2001, 2018

DOI: https://doi.org/10.1200/JC0.18.00630; Published at jco.org on November 8, 2018. 
AUTHORS' DISCLOSURES OF POTENTIAL CONFLICTS OF INTEREST

\section{Reply to V. Turan et al}

The following represents disclosure information provided by authors of this manuscript. All relationships are considered compensated. Relationships are self-held unless noted. I = Immediate Family Member, Inst = My Institution. Relationships may not relate to the subject matter of this manuscript. For more information about ASCO's conflict of interest policy, please refer to www.asco.org/rwc or ascopubs.org/jco/site/ifc.

Matteo Lambertini

Consulting or Advisory Role: TEVA Pharmaceuticals Industries

Lucia Del Mastro

Honoraria: Roche, Pfizer, Ipsen, Eli Lilly, Eisai, Novartis, Takeda Pharmaceuticals

Consulting or Advisory Role: Eli Lilly, Roche, MSD

Travel, Accommodations, Expenses: Roche, Pfizer

No other potential conflicts of interest were reported. 